\title{
Acceso de las mujeres a cargos gerenciales en empresas argentinas socialmente responsables
}

\author{
Estefanía Solari * \\ https://orcid.org/0000-0002-2532-5034
}

Recibido: 14 de mayo de 2020 • Aceptado: 4 de febrero de 2021

\section{Resumen}

La siguiente investigación tuvo como objetivo analizar la participación de las mujeres en puestos gerenciales en empresas socialmente responsables y determinar si se presentan diferencias según el tamaño de la organización. La población objeto de la investigación consistió en 43 reportes de sostenibilidad del periodo 2018-2019 obtenido de empresas argentinas. Se efectuó un análisis descriptivo con posterior tratamiento cuantitativo de los datos. Los principales hallazgos han sido: baja presencia de mujeres en puestos gerenciales $(26.18 \%) \mathrm{y}$, respecto al tamaño de la empresa, mayor participación de mujeres en puestos gerenciales en empresas medianas $(27.35 \%)$ que en empresas grandes $(25.41 \%)$; no obstante, la diferencia no resultó estadísticamente significativa.

Palabras clave: Responsabilidad Social Empresarial, GRI, género, segregación vertical.

* Doctora en Ciencias de la Administración, de la Universidad Nacional de La Plata (UNLP), Argentina. Magíster en Dirección de Empresas de la UNLP. Contadora Público y licenciada en Administración de la UNLP. Docente en grado y posgrado de la UNLP. Integrante de proyectos de investigación desde el 2009. Directora del proyecto de investigación sobre Responsabilidad Social Empresarial desde el 2016, del Instituto de Investigaciones Administrativas de la Facultad de Ciencias Económicas de la UNLP. Correo: estefaniasolari@gmail.com 


\title{
Women's access to management positions in socially responsible Argentinian companies
}

\begin{abstract}
The following research aimed to analyze the participation of women in management positions in socially responsible companies and determine if there are differences according to the size of the organization. The target population consisted of 43 sustainability reports from the period 2018-2019 obtained from Argentinian companies. A descriptive analysis was carried out with subsequent quantitative treatment of the data. The main findings have been: low presence of women in management positions $(26.18 \%)$ and, with regard to the size of the company, greater participation of women in management positions in medium-sized companies $(27.35 \%)$ than in large companies $(25.41 \%)$; however, the difference was not statistically significant.
\end{abstract}

Keywords: Corporate Social Responsibility, GRI, gender, vertical segregation.

\section{L'accès des femmes aux postes de directions dans les entreprises socialement responsables}

\section{Résumé}

Cette étude avait pour objectif d'analyser la participation des femmes dans les entreprises socialement responsables et de déterminer s'il y a des différences selon leur taille. La population objet de la recherche a été 43 rapports de durabilité de la période 2018-2019 des entreprises argentines. Ces rapports ont été analysés de manière descriptive et ultérieurement, les données ont été traités de façon quantitative. Les principales découvertes ont été : faible présence des femmes aux postes de direction $(21.18 \%)$ et, à l'égard de la taille de l'entreprise, il y a plus de participation des femmes aux postes de direction dans les moyennes entreprises $(27.35 \%)$ que dans les grandes entreprises $(25.41 \%)$; cependant, la différence n'est pas statistiquement significative.

Mots-clés: responsabilité sociale des entreprises, GRI, genre, ségrégation verticale. 


\section{Introducción}

Durante la Revolución Industrial se configuró una estructura de clases sociales organizada en torno a la ocupación del sistema productivo, en el cual preponderaba el desempeño de los hombres, quienes asignaban la posición de clase al resto de los miembros de la familia. En la década de 1960, esto se ve modificado ante la progresiva incorporación de las mujeres al mercado laboral remunerado y al sistema de estudios universitarios ${ }^{1}$. No obstante, las mujeres se introducen en el trabajo remunerado con una identidad de género vinculada con el rol de cuidadora y ejecutora de las tareas domésticas ${ }^{2}$, por lo que no se puede dejar de lado la segmentación y segregación del mercado laboral al cual acceden las mujeres.

La investigación de Jiménez-Vargas ${ }^{3}$ presenta que la mayor formación por parte de las mujeres generaría una trasformación del mercado laboral y de su oferta; no obstante, aunque existen indicadores que vislumbran una evolución positiva a favor de la incorporación de las mujeres al mercado laboral, lo cierto es que los datos no reflejan una feminización, ya que esta, solo se ha producido en algunos sectores como el administrativo, o bien, en trabajos como auxiliares, cajeras, recepcionistas, dependientas de comercio o restauración, limpieza etc.; mientras que los hombres ocupan los puestos de dirección o alta dirección, trabajos en empresas de la construcción o en empresas del sector automotriz, así como trabajos en la docencia universitaria, entre otros.

A pesar de la realidad planteada anteriormente, no se puede dudar de la necesaria existencia de mujeres en el Gobierno Corporativo de las empresas, el argumento lógico que lo sugiere está relacionado con la diversidad que la mujer aporta al gobierno de las empresas; a su vez, desde el punto de vista de la comunicación interna y externa, la existencia de una mayor igualdad de género servirá como estrategia de atracción de talento, mejorará la reputación corporativa y será un elemento de política de la Responsabilidad Social Empresarial (RSE) ${ }^{4}$.

Los objetivos de esta investigación se basan en el establecimiento del tamaño y sector de empresas, así como en la especificación de la cantidad de mujeres en puestos gerenciales, para posteriormente determinar si el acceso de mujeres a puestos gerenciales depende del tamaño de la empresa; además, se

\footnotetext{
${ }^{1}$ Mercedes Alcañiz-Moscardó, «Género con clase: la conciliación desigual de la vida laboral y familiar», Revista Española de Sociología, n. ${ }^{\circ} 23$ (2015): 29-55.

2 Jeffrey Neilson y María Stanfors, «It's About Time! Gender, Parenthood, and Household Division of Labor Under Different Welfare Regimes», Journal of Family Issues 35, n. ${ }^{\circ}$ (2014): 1066-1088, doi.org/10.1177/0192513X14522240

${ }^{3}$ Pedro Jesús Jiménez-Vargas, «La desigualdad de género en el mercado laboral», Revista de Información Laboral, n. ${ }^{\circ} 6$ (2017): 65-77.

${ }^{4}$ Irma Martínez-García, María Sacristán-Navarro y Silvia Gómez-Ansón, «Diversidad de género en los consejos de administración: El efecto de la normativa en la presencia de mujeres en las empresas españolas cotizadas», aDResearch ESIC 22, n. ${ }^{\circ} 22$ (2020): 60-81.
} 
parte de la hipótesis de que a mayor tamaño de la empresa, mayor acceso de las mujeres a cargos gerenciales ${ }^{5} 67$. Para probar esta tesis, se efectuó un test de diferencia de medias para muestras independientes. Cabe destacar que no existen investigaciones que hayan estudiado la temática en empresas argentinas.

El artículo está divido en seis secciones: esta primera correspondiente a la introducción, posteriormente se da un fundamento teórico en el cual se expone sobre la diversidad de género y su relación con la RSE, se desarrollan las directrices de la Global Reporting Initiative (GRI) y finalmente se menciona literatura previa sobre la relación entre la implementación de RSE y el tamaño organizacional. Una tercera sección presenta la metodología integrada por: el diseño, el tipo de muestra seleccionado y el método de análisis para probar la hipótesis. En la cuarta se muestran los resultados obtenidos para posteriormente presentar las conclusiones y por último las referencias bibliográficas.

\section{Marco teórico}

\section{Diversidad de género y RSE}

Como se explica a continuación, las temáticas de género están vinculadas con la RSE debido a que esta se va definiendo en torno a tres principales dimensiones: derechos humanos, derechos laborales y medio ambiente ${ }^{8}$; con base en estas, se vuelven de gran relevancia las políticas de género para las primeras dos dimensiones.

Desde su surgimiento, la RSE se ha conceptualizada de diversas maneras; se destaca la manera en la cual Moreno-Izquierdo ${ }^{9}$ la define, como: «la libre voluntad de la empresa de aportar el mayor valor posible a sus diferentes grupos de interés». De esta se desprenden las siguientes ideas: a) se trata de una voluntad no obligada por imperativo legal, si bien la RSE bien entendida exige un escrupuloso cumplimiento de la ley, las buenas prácticas a que da lugar deben rebasar las exigencias legales (sino se estaría ante la simple

\footnotetext{
${ }^{5}$ Nuria Bajo-Davó y Juan José Durán-Herrera, «Responsabilidad social y variables estratégicas en las grandes empresas españolas», Revista de Responsabilidad Social de la Empresa, n. ${ }^{\circ} 2$ (2009): 51-76.

${ }^{6}$ Alberto Lafuente, Víctor Viñuales, Ramón Pueyo y Jesús Llaría, Responsabilidad social corporativa y políticas públicas (Madrid: Fundación Alternativas, 2003).

${ }^{7}$ Antonio Vives y Estrella Peinado-Vara, «La responsabilidad social de la empresa en América Latina» (2011), acceso: 1 de junio de 2021,

https://publications.iadb.org/publications/spanish/document/La-responsabilidad-social-de-la-empresaen-América-Latina-Manual-de-Gestión.pdf

${ }^{8}$ Paul Capriotti y Francisco José Garrido, Responsabilidad Social Empresaria. Hacia una reputación sustentable para el siglo XXI (Barcelona: EXEC Business School Publishing, 2018).

${ }^{9}$ Moreno-Izquierdo, José Ángel, «Responsabilidad social corporativa y competitividad: una visión desde la empresa», Revista Valenciana de Economía y Hacienda, n. ${ }^{\circ} 2$ (2004): 9-49.
} 
responsabilidad legal); b) en segundo lugar, esa voluntad de aportación de valor a cada parte interesada está condicionada por la paralela voluntad de tratar con criterio similar a los restantes; y c) se trata de un principio que conduce a una tercera implicación: esa atención mesurada a todos los grupos de interés supone tomar en consideración todas las dimensiones de la actividad de la empresa.

Cabe aclarar que cuando se pretende crear «valor» para cada parte interesada, se asume la postura de Argandoña ${ }^{10}$, quien considera que el concepto de valor va más allá del valor extrínseco de naturaleza económica para incluir otros tipos de valores que las partes interesadas también necesitan, estos son: valores extrínsecos inmateriales que proporciona la empresa (por ejemplo, algún tipo de formación), valores intrínsecos psicológicos (como la satisfacción por el trabajo realizado) y valores intrínsecos operativos (como podría ser la adquisición de conocimiento o capacidades).

Dentro del ámbito interno de la RSE las personas trabajadoras ocupan un lugar destacado e importante en materia de la gestión de los recursos humanos. Este debe contribuir con la realización de buenas prácticas antes de la contratación, durante y después de ella, y en general en temas como: formación, desarrollo profesional, igualdad de género, igualdad salarial, cualificación profesional, conciliación de la vida laboral, familiar y personal, seguridad en el trabajo, socialización, prácticas responsables de contratación, acceso de minorías a empleos dignos y con calidad, entre otras, con las que la empresa debe velar por la permanencia de sus colaboradoras y colaboradores, así como atraer personas cualificadas para su desarrollo profesional y personal, incidiendo en el progreso de una sociedad local, que recibe y asume de forma directa o indirecta las consecuencias de sus actuaciones ${ }^{11}$.

En el escenario de la RSE, la promoción de la igualdad de la mujer debe darse en cada uno de los roles que esta desempeña en los grupos de interés, tales como: empleadas, consumidoras, integrantes de la comunidad, proveedoras, participantes de las organizaciones civiles, inversionistas, entre otros. En contraposición, la inequidad de género se manifiesta de múltiples formas en el lugar de trabajo: reclutamiento, desarrollo profesional y promoción, salarios y segregación laboral (vertical y horizontal) ${ }^{12}$.

\footnotetext{
${ }^{10}$ Antonio Argandoña, La teoría de los stakeholders y la creación de valor (Barcelona: IESE Business School, 2011).

${ }^{11}$ Isabel Jaramillo-Arango, «Reflexiones acerca de la responsabilidad social empresarial desde su dimensión laboral», Revista Espiga 14, n. ${ }^{\circ} 30$ (2015), 9-22, acceso: 31 de mayo de 2021, https://revistas.uned.ac.cr/index.php/espiga/article/view/926

${ }_{12}$ Yenni Duque-Orozco, Marleny Cardona Acevedo y Jaime Rendón Acevedo, «Responsabilidad Social Empresarial: Teorías, índices, estándares y certificaciones», Cuadernos de Administración 29, n. ${ }^{\circ} 50$ (2014), 196-206, acceso: 31 de mayo de 2021,

https://www.redalyc.org/articulo.oa?id=225029797009
} 
Por lo tanto, al desarrollar prácticas de RSE en el ámbito laboral, no se puede omitir hacer mención de que estas buscarán que no se origine segregación de género, lo cual podría convertirse en una situación discriminatoria en la medida de que las actividades laborales femeninas vayan acompañadas de sueldos más bajos, mayor índice de desempleo, menor valoración social y mayor inestabilidad ${ }^{13}$. A su vez, las empresas socialmente responsables deberían contemplar la importancia respecto de que cualquier organización requiere de un estilo directivo impregnado tanto de valores masculinos como femeninos, aprovechando el aporte que las mujeres altamente cualificadas pueden realizar a las empresas, por lo que se hace de suma importancia introducir un enfoque de diversidad de género en el mundo empresarial ${ }^{14}$.

Otra investigación ${ }^{15}$ resalta la importancia de las mujeres en cargos gerenciales, se demuestra que estas son una fuerza motora de la RSE interna, por lo que concluyen que las empresas a las cuales les interese fortalecer sus prácticas de RSE, deben considerar, en particular, el género de la persona que dirige la organización porque va a influir en la gestión. Al analizar la relación entre la participación de mujeres en cargos gerenciales y la adopción de prácticas de RSE, resultados empíricos han demostrado que, a mayor diversidad de género, factores como la rentabilidad, el desempeño ambiental y social de la empresa se incrementan ${ }^{16}$.

No obstante, los beneficios generados por el hecho de que cargos directivos estén ocupados por mujeres en los más altos niveles jerárquicos organizacionales, se evidencia la casi nula presencia de estas en la alta dirección de las compañías ${ }^{17}$; así, aún existen empresas que contemplan la RSE como no ajenas de los efectos de la segregación vertical, es decir, en estas organizaciones, la toma de decisiones, no necesariamente cuenta con la participación igualitaria de hombres y mujeres ${ }^{18}$.

\footnotetext{
${ }^{13}$ Maite Sarrió, Amparo Ramos, Esther Barberá, Carlos Candela, «Más allá del techo de cristal», Revista del Ministerio de Trabajo y Asuntos Sociales: Revista del Ministerio de Trabajo e Inmigración, $\mathrm{n}^{\circ} 40$ (2002): 55-68.

${ }^{14}$ Nina Aguiar y Deolinda Meira, «Study on the efficacy of the Portuguese cooperative taxation», REVESCO Revista de Estudios Cooperativos 121 (2015): 7-32, acceso: 31 de mayo de 2021, https://www.researchgate.net/publication/311640466_Study_on the efficacy_of the Portuguese_co operative taxation

${ }^{15}$ Florina Guadalupe Arredondo-Trapero, Luz María Velázquez-Sánchez y Jorge de la Garza García, «Políticas de diversidad y flexibilidad laboral en el marco de la responsabilidad social empresarial. Un análisis desde la perspectiva de género», Estudios Gerenciales 29, n. ${ }^{\circ} 127$ (2013): 161-166.

${ }^{16}$ Carolina Reyes-Bastidas, Guadalupe del Carmen Briano-Turrent y María Luisa Saavedra-García, «Diversidad de género en el consejo y su incidencia en la responsabilidad social en empresas cotizadas de Colombia y México», Contaduría y Administración 65, n. ${ }^{\circ} 3$ (2020): 1-27, doi.org/10.22201/fca.24488410e.2020.2241

${ }^{17}$ Orencio Vázquez-Oteo y Carlos Cordero-Sanz, «Análisis empírico de la información emitida por las empresas del IBEX 35 en materia de responsabilidad social corporativa», Ekonomiaz: Revista Vasca de Economía, n. ${ }^{\circ} 65$ (2007): 150-183.

${ }^{18}$ María Martínez León, Narciso Arcas León y Margarita García Hernández, La influencia del género sobre la responsabilidad social empresarial en las entidades de economía social, REVESCO. Revista de Estudios Cooperativos (105), 143-172.
} 


\section{Global Reporting Initiative (GRI) y la equidad de género}

Según la GRI, la elaboración de reportes de sostenibilidad ayuda a las organizaciones a marcarse objetivos, medir el desempeño y gestionar el cambio con el propósito de que sus operaciones sean más sostenibles. Los reportes de sostenibilidad contienen información sobre la incidencia de las organizaciones, ya sea positiva o negativa, en el medio ambiente, la sociedad y la economía. También, los reportes contienen indicadores para el seguimiento de las prácticas de RSE para llevar a cabo el control adecuado. Otro aporte sustancial de los reportes de sostenibilidad se origina al acordar, a nivel internacional, los contenidos y los sistemas de medida, esto favorece la accesibilidad y la comparabilidad y, por consiguiente, mejora la calidad de la información para que los grupos de interés tomen las decisiones pertinentes ${ }^{19}$.

El marco para la elaboración de reportes de sostenibilidad de la GRI se creó para facilitar un lenguaje común que pudiera ser aplicado por todo tipo de organizaciones y se ha redactado con referencia a un gran número de acuerdos y normas internacionales. Cientos de personas y de organizaciones que integran la red de la GRI han descubierto que el proceso de elaboración de reportes, conforme sus directrices, son la mejor manera de comprender y cuantificar el alcance de la gestión de la $\mathrm{RSE}^{20}$.

Respecto de la elaboración de los reportes de sostenibilidad, en primer lugar, la GRI propuso la Guía G3, en la cual se les permitía a las empresas elegir el nivel en el que querían elaborar los reportes (desde C, para los principiantes, hasta $\mathrm{A}+$ para los más experimentados), reflejando los diferentes grados de utilización del Marco de la GRI. Luego la GRI elaboró la Guía G4, así como todos los reportes que se publicaron después del 31 de diciembre de 2015, los cuales debieron confeccionarse de conformidad con ella.

La Guía G4 establece que aquellas organizaciones que deseen elaborar sus reportes de sostenibilidad, de conformidad con la Guía, tienen dos opciones: la opción Esencial y la opción Exhaustiva ${ }^{21}$. La opción Esencial consta de los elementos fundamentales de un reporte de sostenibilidad, constituye un marco mediante el cual las organizaciones transmiten las consecuencias de su desempeño económico, ambiental, social y de gobierno; mientras que la opción Exhaustiva desarrolla la Esencial (describiendo su desempeño de un modo más pormenorizado), incorporando nuevos contenidos básicos relativos a la estrategia, el análisis, el gobierno, la ética y la integridad de las organizaciones $^{22}$.

\footnotetext{
${ }^{19}$ Global Reporting Initiative (GRI), Guía para la elaboración de memorias de sostenibilidad. Manual de aplicación (Amsterdam: GRI, 2013).

${ }^{20}$ Global Reporting Initiative (GRI), Guía para...

${ }^{21}$ Global Reporting Initiative (GRI), Guía para ...

${ }^{22}$ Global Reporting Initiative (GRI), Guía para... 
Los indicadores propuestos por la GRI ofrecen información sobre el desempeño o sobre los efectos económicos, ambientales y sociales de la organización en referencia de sus aspectos materiales. Los aspectos materiales son aquellos que reflejan los impactos económicos, ambientales y sociales significativos de la organización, o bien, aquellos que tienen un peso notable en las evaluaciones y decisiones de los grupos de interés. Estos grupos de interés están constituidos por accionistas, empleados, proveedores, el Estado y la sociedad en su conjunto ${ }^{23}$.

Los indicadores propuestos por la GRI se agrupan en tres dimensiones: dimensión económica, dimensión ambiental y la dimensión social, compuesta a su vez por cuatro subdimensiones: Prácticas Laborables y Ética en el Trabajo, Derechos Humanos, Prácticas Sociales y Responsabilidad sobre Productos.

Para cada dimensión, la GRI propone el seguimiento de un conjunto de indicadores, incluyendo la subdimensión Prácticas Laborables y Ética en el Trabajo, en el indicador: «Composición de los órganos de gobierno corporativo y plantilla, desglosado por sexo, grupo de edad, pertenencia al grupo minoritario y otros indicadores de diversidad», en el que se centra la presente investigación.

\section{Implementación de prácticas de RSE y su relación con el tamaño organizacional}

Las prácticas de RSE deben hacerse extensivas a todo tipo de empresas, con independencia de su tamaño y localización. En este mismo sentido, empresarios PYMES manifestaron como muy relevante el comprender que la RSE y el cumplimiento de los Principios Rectores no son una exclusividad de las grandes empresas, sobre las que se ha puesto el foco, sino de todas las empresas, con independencia de su tamaño, sector o geografía ${ }^{24}$.

No obstante lo mencionado en el párrafo anterior, distintos autores han evidenciado que el tamaño de la empresa podría influir en la intensidad con que se implementan las prácticas de RSE. De esta manera, Lafuente ${ }^{25} \mathrm{y}$ Vives $^{26}$ constataron una correlación positiva entre un mayor tamaño y un mayor desarrollo de RSE en la empresa española. Bajo-Davó y DuránHerrera $^{27}$ se alinean con la idea anterior, al demostrar que en las empresas

\footnotetext{
${ }^{23}$ María del Mar Alonso-Almeida, Martha del Pilar Rodríguez-García, Klender Aimer CortezAlejandro y José Luis Abreu-Quintero, «La responsabilidad social corporativa y el desempeño financiero: análisis en empresas mexicanas que cotizan en la bolsa», Contaduría y Administración 57, n. ${ }^{\circ} 1$ (2012): 53-77.

${ }^{24}$ Bernardo Cruza-Martos, «El compromiso activo de las empresas con los derechos humanos», Tiempo de paz, n. ${ }^{\circ} 122$ (2016): 46-52.

${ }^{25}$ Alberto Lafuente, Víctor Viñuales, Ramón Pueyo y Jesús Llaría, Responsabilidad social...

${ }^{26}$ Antonio Vives y Estrella Peinado-Vara, «La responsabilidad social...

${ }^{27}$ Nuria Bajo-Davó y Juan José Durán-Herrera, «Responsabilidad social...
} 
englobadas dentro de los grupos de mayor tamaño, se evidencian mayores niveles de actividad en RSE. Chivite-Cebolla, Enciso de Yzaguirre, GarcíaOsma y Túa-Pereda ${ }^{28}$ presentan resultados similares, obteniendo entre sus hallazgos que el tamaño de la empresa, su endeudamiento y el sector de actividad influyen en la aplicación de la RSE.

\section{Desarrollo}

\section{Metodología}

Del total de reportes presentados por las empresas argentinas adheridas a la GRI durante el periodo 2018-2019 (un total de 133), se seleccionó los 43 reportes que informaban sobre el indicador GRI: Composición de los órganos de gobierno corporativo y plantilla, desglosado por sexo, grupo de edad, pertenencia a grupo minoritario y otros indicadores de diversidad; por lo tanto el muestreo fue de tipo intencional, puesto que las unidades de análisis se eligieron por reunir ciertas características representativas del fenómeno objeto de estudio.

El tipo de diseño es no experimental, no se manipulan de manera intencional una o más variables independientes (causas) para analizar las consecuencias sobre una o más variables dependientes (efectos). La investigación es de tipo transversal, ya que se observan las características de la población objeto de estudio en un solo momento temporal y no se pretende relevar el comportamiento de las variables en el tiempo.

Con base en la información publicada en los reportes de sostenibilidad, específicamente en relación al indicador: Composición de los órganos de gobierno corporativo y plantilla, desglosado por sexo, grupo de edad, pertenencia a grupo minoritario y otros indicadores de diversidad, se calculó para cada empresa el siguiente ratio:

Proporción de Mujeres que ocupan cargos gerenciales $=($ total de mujeres en puesto gerenciales/total de gerentes) $\times 100$

En cuanto a los métodos de análisis, en primer lugar se desarrolló una serie de tablas de frecuencia y luego, con base en los hallazgos de Bajo-Davó y Durán-Herrera $^{29}$, Lafuente ${ }^{30}$ y Vives ${ }^{31}$ se planteó contrastar la hipótesis de: $a$ mayor tamaño de la empresa, mayor acceso de las mujeres a cargos

\footnotetext{
${ }^{28}$ María Peana Chivite-Cebolla, Vicente Enciso de Yzaguirre, Beatriz García-Osma y Jorge TúaPereda, «Determinantes de la Responsabilidad Social Corporativa: la crisis y el efecto contagio», CIRIEC - España. Revista de economía pública social y cooperativa, n. ${ }^{\circ} 81$ (2014): 127-162.

${ }^{29}$ Nuria Bajo-Davó y Juan José Durán-Herrera, «Responsabilidad social...

${ }^{30}$ Alberto Lafuente, Víctor Viñuales, Ramón Pueyo y Jesús Llaría, Responsabilidad social...

${ }^{31}$ Nuria Bajo-Davó y Juan José Durán-Herrera, «Responsabilidad social... 
gerenciales, para lo cual se procedió a realizar un análisis de Diferencia de medias para muestras independientes, debido a que la proporción de cargos gerenciales ocupados por mujeres se pueden clasificar en función de la variable dicotómica tamaño (que toma los siguientes valores Empresas grandes $=1$ y Empresas medianas $=0$ ). De esta manera se buscó contrastar la siguiente hipótesis nula:

$$
\text { Ho: } \mu_{\text {empresas grandes }}=\mu_{\text {empresas medianas }}
$$

Cabe aclarar que la variable tamaño fue considerada según la definición que utiliza la GRI para esta clasificación, que según la Unión Europea se define como: empresa mediana a aquella con un rango entre 50-249 empleados, un volumen de facturación $<=50$ millones de euros o activo $<=43$ millones; mientras que empresas grandes son aquellas con más de 250 empleados y un volumen de facturación $>50$ millones de euros o activo $>43$ millones.

\section{Resultados}

En este apartado, en una primera parte se expondrán las características (tamaño y sector) de las empresas cuyos reportes fueron objeto de la investigación, en una segunda parte se expondrán los resultados concernientes al acceso de las mujeres a los cargos gerenciales y posteriormente se expone la discusión de los resultados.

\section{Características de la población objeto de la investigación}

En primer lugar, respecto de la caracterización de la población, esta estuvo compuesta por 43 empresas, las grandes corresponden al $60.5 \%$, mientas que las medianas al $39.5 \%$ (Tabla 1 ).

Tabla 1. Cantidad de empresas según tamaño

\begin{tabular}{|l|c|c|}
\hline \multicolumn{1}{|c|}{ Tamaño } & Cantidad & Porcentaje \\
\hline Grandes & 26 & 60.5 \\
\hline Medianas & 17 & 39.5 \\
\hline Total & 43 & 100 \\
\hline
\end{tabular}

Fuente: elaboración propia.

En cuanto al sector de actividad al cual pertenecen (Tabla 2), se destaca el sector de Productos alimenticios y bebidas (16.3\%), seguido de Servicios financieros (14 \%) y Logística y Servicios comerciales con un $10.4 \%$. 
Tabla 2. Cantidad de empresas según sector de actividad

\begin{tabular}{|l|c|c|}
\hline \multicolumn{1}{|c|}{ Sector } & Cantidad & Porcentaje \\
\hline Productos alimenticios y bebidas & 7 & 16.3 \\
\hline Servicios financieros & 6 & 14.0 \\
\hline Logística & 5 & 11.6 \\
\hline Servicios comerciales & 5 & 11.6 \\
\hline Energía & 3 & 7.0 \\
\hline Medios & 3 & 7.0 \\
\hline Servicios de energía & 3 & 7.0 \\
\hline Tecnológico & 3 & 7.0 \\
\hline Minería & 2 & 4.7 \\
\hline Servicios de salud & 2 & 4.7 \\
\hline Agricultura & 1 & 2.3 \\
\hline Automotriz & 1 & 2.3 \\
\hline Productos para la salud & 1 & 2.3 \\
\hline Telecomunicaciones & 1 & 2.3 \\
\hline Otros & 43 & 100 \\
\hline
\end{tabular}

Fuente: elaboración propia.

\section{Acceso de las mujeres a cargos gerenciales}

Conforme con lo establecido en los objetivos, y según la metodología planteada, en la tabla 3 se detallan estadísticas descriptivas de la variable «proporción de cargos gerenciales ocupados por mujeres», de las empresas analizadas, en promedio, las mujeres que ocupan cargos gerenciales representan un $26.18 \%$, con un desvío de $13.1 \%$, así como un valor mínimo de $4 \%$ y un valor máximo de $53 \%$.

Tabla 3. Estimadores descriptivos de la variable «proporción de cargos gerenciales ocupados por mujeres»

\begin{tabular}{|l|c|c|c|c|c|}
\hline & Obs. & Media & Desvío & Mínimo & Máximo \\
\hline $\begin{array}{l}\text { Proporción de cargos gerenciales } \\
\text { ocupados por mujeres }\end{array}$ & 43 & 26.18 & 13.1 & 4 & 53 \\
\hline
\end{tabular}

Fuente: elaboración propia.

Luego, para determinar si el acceso de mujeres a puestos gerenciales depende del tamaño de la empresa, se efectuó el test de diferencia de medias para muestras independientes para contrastar la siguiente hipótesis: a mayor tamaño de la empresa mayor acceso de las mujeres a cargos gerenciales.

En la tabla 4 se observa que la media de la proporción de cargos gerenciales ocupados por mujeres es de $25.41 \%$ en el caso de empresas grandes y de $27.35 \%$ en las medianas, se evidencia mayor participación de mujeres en las empresas medianas que en las grandes, aunque no resulta una diferencia 
estadísticamente significativa (a un nivel de significación de $95 \%$ ), por lo que no se rechaza la hipótesis nula, no se evidencia diferencias significativas, para la población objeto de la investigación, en relación con la participación de las mujeres en cargos gerenciales según el tamaño de la empresa.

Tabla 4. Diferencia de medias de la proporción de cargos gerenciales ocupados por mujeres por tamaño de la empresa

\begin{tabular}{|l|c|c|}
\hline \multicolumn{1}{|c|}{ Tamaño empresas } & Obs. & $\begin{array}{c}\text { Media }(\mu) \\
\text { Proporción de cargos } \\
\text { gerenciales ocupados por } \\
\text { mujeres }\end{array}$ \\
\hline Grandes & 26 & 25.41 \\
\hline Medianas & 17 & 27.35 \\
\hline $\begin{array}{l}\text { Diferencia: } \\
\mu \text { Empresas grandes- } \mu \text { Empresas medianas }\end{array}$ & $3.18 \#$ \\
\hline
\end{tabular}

Nota:\# p-valor $>0.05$

Fuente: elaboración propia.

\section{Discusión de los resultados}

Mediante los resultados, se evidencia una baja participación de las mujeres en cargos gerenciales de las empresas analizadas, es decir, aun tratándose de empresas socialmente responsables que deberían contemplar la importancia respecto a la incorporación de la diversidad de género, la participación de las mujeres no es alta. En cuanto al tamaño organizacional, tampoco se observa que en las empresas más pequeñas, con menos jerarquías, la incorporación de diversidad de género en los cargos gerenciales sea mayor que en las grandes empresas.

Esto deja de manifiesto que, aún en las empresas socialmente responsables, debe seguir profundizándose su accionar en pos de la diversidad de género que permita el desarrollo profesional, tanto a hombres como a mujeres, y en provecho del aporte que las mujeres altamente cualificadas pueden realizar a las empresas.

\section{Conclusiones}

La principal conclusión es que se evidenció, para el total de las empresas socialmente responsables analizadas, que solo un $26.18 \%$ de los cargos gerenciales son ocupados por mujeres, cuando la GRI y diversos organismos promotores de la RSE consideran fundamental la equidad de género en los distintos niveles organizacionales; por lo que no se estaría contemplando lo 
que han evidenciado investigaciones como las de Arredondo-Trapero ${ }^{32}$, quienes resaltan la importancia de las mujeres en cargos gerenciales, puesto que demostraban una fuerza motora de la RSE interna. Los hallazgos de la presente investigación se alinean con los de y Martínez-León ${ }^{33}$, evidenciándose que incluso las empresas que contemplan la RSE, no son ajenas a los efectos de la segregación vertical.

Respecto de la hipótesis a mayor tamaño de la empresa mayor acceso de las mujeres a cargos gerenciales, los resultados se contraponen a los hallazgos de Bajo-Davó y Durán-Herrera ${ }^{34}$, Lafuente ${ }^{35}$ y Vives ${ }^{36}$ se presentó mayor presencia en puestos gerenciales de mujeres en las empresas medianas versus las grandes $(27.35 \%$ contra $25.41 \%$ ), al margen de que la diferencia no resultó estadísticamente significativa ( $\mathrm{p}$-valor $>0.05$ ), quedó en evidencia cómo las empresas con menor jerarquías estarían adaptándose mejor a los desafíos que se propone a las empresas socialmente responsables respecto de la incorporación de las mujeres en cargos gerenciales.

Por todo lo expuesto, la desigualdad de género es una realidad difícil de erradicar, lo cual la convierte en un desafío, incluso para aquellas empresas que llevan a cabo su gestión conforme la RSE. Este es un tema en el que queda mucho por avanzar y en el cual se debe continuar la promoción de políticas en pos de la igualdad de género. Asimismo, serán la formación y la educación, desde los niveles iniciales, las claves para conseguir la tan deseada igualdad en el más amplio sentido, no solo la igualdad de género.

El presente estudio, al ser de tipo exploratorio, permitió obtener una primera aproximación del tema abordado, por lo que se considera pertinente aclarar la intención de profundizar el análisis en futuras investigaciones, ampliando el número de reportes de sustentabilidad relevados y con la concertación de entrevistas en profundidad con algunos empresarios, esto para enriquecer los resultados y las causales que llevan a las empresas socialmente responsables a seguir contando con baja participación de mujeres en puestos gerenciales.

A su vez, en el ámbito académico, este trabajo puede ser un referente para continuar investigando sobre la forma en la cual la diversidad y la flexibilidad son contempladas en las prácticas laborales de empresas socialmente responsables.

\footnotetext{
${ }^{32}$ Florina Guadalupe Arredondo-Trapero, Luz María Velázquez-Sánchez y Jorge de la Garza García, «Políticas de...

${ }^{33}$ María Martínez León, Narciso Arcas León y Margarita García Hernández, La influencia del género...

${ }^{34}$ Nuria Bajo-Davó y Juan José Durán-Herrera, «Responsabilidad social...

${ }^{35}$ Alberto Lafuente, Víctor Viñuales, Ramón Pueyo y Jesús Llaría, Responsabilidad social...

${ }^{36}$ Antonio Vives y Estrella Peinado-Vara, «La responsabilidad social...
} 


\section{Formato de citación según APA}

Solari, E. (2021). Acceso de las mujeres a cargos gerenciales en empresas argentinas socialmente responsables. Revista Espiga, 21 (41), páginas 121-136.

\section{Formato de citación según Chicago-Deusto}

Solari, Estefanía. «Acceso de las mujeres a cargos gerenciales en empresas argentinas socialmente responsables». Revista Espiga 21, n. ${ }^{\circ} 41$ (enero-junio, 2021): páginas 121-136.

\section{Referencias}

Aguiar, Nina y Deolinda Meira. «Study on the efficacy of the Portuguese cooperative taxation». REVESCO Revista de Estudios Cooperativos 121 (2015): 7-32. Acceso: 31 de mayo de 2021. https://www.researchgate.net/publication/311640466 Study on the efficacy o $\mathrm{f}$ the Portuguese cooperative taxation

Alcañiz-Moscardó, Mercedes. «Género con clase: la conciliación desigual de la vida laboral y familiar». Revista Española de Sociología, n. ${ }^{\circ} 23$ (2015): 29-55.

Alonso-Almeida, María del Mar, Martha del Pilar Rodríguez-García, Klender Aimer Cortez-Alejandro y José Luis Abreu-Quintero. «La responsabilidad social corporativa y el desempeño financiero: análisis en empresas mexicanas que cotizan en la bolsa». Contaduría y Administración 57, n. ${ }^{\circ}$ (2012): 53-77.

Argandoña, Antonio. La teoría de los stakeholders y la creación de valor. Barcelona: IESE Business School, 2011.

Arredondo-Trapero, Florina Guadalupe, Luz María Velázquez-Sánchez y Jorge de la Garza García. «Políticas de diversidad y flexibilidad laboral en el marco de la responsabilidad social empresarial. Un análisis desde la perspectiva de género». Estudios Gerenciales 29, n. ${ }^{\circ} 127$ (2013): 161-166.

doi.org/10.1016/j.estger.2013.05.004

Bajo-Davó, Nuria y Juan José Durán-Herrera. «Responsabilidad social y variables estratégicas en las grandes empresas españolas». Revista de Responsabilidad Social de la Empresa, n. 2 (2009): 51-76.

Capriotti, Paul y Francisco José Garrido. Responsabilidad Social Empresaria. Hacia una reputación sustentable para el siglo XXI. Barcelona: EXEC Business School Publishing, 2018. 
Chivite-Cebolla, María Peana, Vicente Enciso de Yzaguirre, Beatriz García-Osma y Jorge Túa-Pereda. «Determinantes de la Responsabilidad Social Corporativa: la crisis y el efecto contagio». CIRIEC - España. Revista de economía pública social y cooperativa, $\mathrm{n} .^{\circ} 81$ (2014): 127-162.

Cruza-Martos, Bernardo. «El compromiso activo de las empresas con los derechos humanos». Tiempo de paz, n. ${ }^{\circ} 122$ (2016): 46-52.

Duque-Orozco, Yenni, Marleny Cardona Acevedo y Jaime Rendón Acevedo. «Responsabilidad Social Empresarial: Teorías, índices, estándares y certificaciones». Cuadernos de Administración 29, n. 50 (2014), 196-206. Acceso: 31 de mayo de 2021. https://www.redalyc.org/articulo.oa?id=225029797009

Global Reporting Initiative (GRI). Guía para la elaboración de memorias de sostenibilidad. Manual de aplicación. Amsterdam: GRI, 2013.

Jaramillo-Arango, Isabel. «Reflexiones acerca de la responsabilidad social empresarial desde su dimensión laboral». Revista Espiga 14, n. ${ }^{\circ} 30$ (2015), 922. Acceso: 31 de mayo de 2021. https://revistas.uned.ac.cr/index.php/espiga/article/view/926

Jiménez-Vargas, Pedro Jesús. «La desigualdad de género en el mercado laboral». Revista de Información Laboral, n. ${ }^{\circ} 6$ (2017): 65-77.

Lafuente, Alberto, Víctor Viñuales, Ramón Pueyo y Jesús Llaría. Responsabilidad social corporativa y politicas públicas. Madrid: Fundación Alternativas, 2003.

Martínez León, María, Narciso Arcas León y Margarita García Hernández. La influencia del género sobre la responsabilidad social empresarial en las entidades de economía social. REVESCO. Revista de Estudios Cooperativos (105). 143-172.

Martínez-García, Irma, María Sacristán-Navarro y Silvia Gómez-Ansón. «Diversidad de género en los consejos de administración: El efecto de la normativa en la presencia de mujeres en las empresas españolas cotizadas». aDResearch ESIC 22, n. ${ }^{\circ} 22$ (2020): 60-81.

Moreno-Izquierdo, José Ángel. «Responsabilidad social corporativa y competitividad: una visión desde la empresa». Revista Valenciana de Economía y Hacienda, . $^{\circ} 12$ (2004): 9-49.

Neilson, Jeffrey y María Stanfors. «It's About Time! Gender, Parenthood, and Household Division of Labor Under Different Welfare Regimes». Journal of Family Issues 35, n. ${ }^{\circ} 8$ (2014): 1066-1088. doi.org/10.1177/0192513X14522240 
Reyes-Bastidas, Carolina, Guadalupe del Carmen Briano-Turrent y María Luisa Saavedra-García. «Diversidad de género en el consejo y su incidencia en la responsabilidad social en empresas cotizadas de Colombia y México». Contaduría y Administración 65, n. ${ }^{\circ} 3$ (2020): 1-27.

doi.org/10.22201/fca.24488410e.2020.2241

Sarrió, Maite, Amparo Ramos, Esther Barberá, Carlos Candela. «Más allá del techo de cristal». Revista del Ministerio de Trabajo y Asuntos Sociales: Revista del Ministerio de Trabajo e Inmigración, n. ${ }^{\circ} 40$ (2002): 55-68.

Vázquez-Oteo, Orencio y Carlos Cordero-Sanz. «Análisis empírico de la información emitida por las empresas del IBEX 35 en materia de responsabilidad social corporativa». Ekonomiaz: Revista Vasca de Economía, n. ${ }^{\circ} 65$ (2007): 150-183.

Vives, Antonio y Estrella Peinado-Vara. «La responsabilidad social de la empresa en América Latina» (2011). Acceso: 1 de junio de 2021.

https://publications.iadb.org/publications/spanish/document/La-responsabilidadsocial-de-la-empresa-en-América-Latina-Manual-de-Gestión.pdf 